\title{
Hierarchical models of intra-specific competition: scramble versus contest
}

\author{
Shandelle M. Henson and J. M. Cushing * \\ Department of Mathematics, Interdisciplinary Program on Applied Mathematics, \\ University of Arizona, Tucson, AZ 85721, USA
}

Received 30 January 1995; received in revised form 5 June 1995

\begin{abstract}
Hierarchical structured models for scramble and contest intraspecific competition are derived. The dynamical consequences of the two modes of competition are studied under the assumption that both populations divide up the same amount of a limiting resource at equal population levels. A comparison of equilibrium levels and their resiliences is made in order to determine which mode of competition is more advantageous. It is found that the concavity of the resource uptake rate is an important determining factor. Under certain circumstances contest competition is more advantageous for a population while under other circumstances scramble competition is more advantageous.
\end{abstract}

Key words: Structured population - Competition

\section{Introduction}

One way density dependent regulation of population growth can occur is through intra-specific competition for limited resources. By affecting the amount of resource available and therefore consumed by an individual organism, such competition can have a significant effect on the individual's vital growth, fertility, and survival rates. This in turn can have a determining effect on the population's dynamics.

The types of interactions between individuals in a population can be diverse, ranging over all those familiar in the study of interspecific interactions and more [32]. In particular, biologists distinguish a variety of different types of intra-specific competition, including contest, scramble, exploitative, and interference. Because they lack underlying submodels for vital physiological characteristics of individual organisms, classical differential equations for 
population level statistics (such as total population size or density, biomass, dry weight etc.) are generally too qualitative to give an adequate accounting of intra-specific competition. As a result, these kinds of model equations cannot describe differing types of competitive interactions and compare and contrast their dynamical consequences for the population.

Structured population dynamics provide a modeling methodology that bridges the gap between the level of the individual organism and that of the total population. Such models classify members of the population according to relevant physiological characteristics (such as chronological age, body size or weight, life cycle stages, genetic or biochemical composition, etc.) and provide submodels for class specific vital rates. Two broad types of models have been widely used: discrete and continuous models. Both types have been used to study a variety of types of intra-specific interactions, including juvenile versus adult competition $[12-15,4-6,25,29]$; cannibalism $[1,3,7,8,11,18$, 19, 21, 33]; and contest versus scramble competition [22, 23, 9, 10].

In this paper we will use a class of continuous structured models to study intra-specific competition. In particular, we are interested in comparing the dynamical consequences of two opposed types of competition, contest and scramble, in order to see under what circumstance one type might be more "advantageous" for a population than the other, according to a specified criterion. This question is one of the main issues in the book by Lomnicki [22]. After pointing out the confused state of affairs with regard to the meanings of "scramble" and "contest" competition as they are used in the literature, Lomnicki provides a clear definition of these terms and studies their dynamical consequences by means of simple discrete model equations. One of his main conclusions is that contest competition is more advantageous to a population. This question was considered in [9] and [10] using more sophisticated, continuous age- and size-structured models. The results in these papers support Lomnicki's conclusion, in so far as equilibrium level and resilience criteria are concerned.

We have several purposes in this paper. Our main goal is to study further the comparison between contest and scramble intra-specific competition and to address the robustness of Lomnicki's tenet that contest competition is generally more advantageous. There are many ways in which a particular type of intra-specific competition might be considered "advantageous" or "disadvantageous" to a population. In this regard most references in the literature study the stability versus instability of a positive equilibrium. For example, see $[22-24,27]$. We, on the other hand, will study the effct that the competition type has on equilibrium levels and resilience. (In fact, in our general class of models below, the positive equilibrium is always globally stable.)

While we take the approach in [9] and [10], we modify and generalize significant aspects of the models used in these papers. Instead of comparing contest to scramble by means of a homotopy models that connects one to the other, as in [9] and [10], we use here a comparison criterion based upon the total amount of limiting resource available to the populations. Thus, we compare scramble and contest populations that divide up the same total 
amounts of the limiting resource (given identical population sizes). We also allow for nonlinear resource uptake rates (such as Holling type II and III), as opposed to the less realistic linear (Volterra type) uptake rates used in [22], [9] and [10]. As we will see, the concavity of the uptake rate, as a function of available resource, plays a significant role in the sense that it can reverse the comparative advantages of the contest and scramble modes of competition.

Continuous structured models involve complicated nonlinear integropartial differential equations, often with nonlinear integro-boundary conditions, and therefore are generally difficult to analyze mathematically. For this reason simplifying assumptions are necessary. One approach taken by many authors has been to study classes of models that can mathematically be reduced, by means of some trick or other, to more tractable equations, such as ordinary differential equations or integral equations. Examples include socalled "separable equations" [28, 2], "linear chain trickery" [17, 26], and "hierarchically structured" models $[9,10]$. The latter type of models are particularly useful for studying contest and scramble competition because the definitions of these modes of competition involve a hierarchical ranking within the population [22]. Hierarchically age-structured models have been rigorously shown to have total population size dynamics governed by a decoupled ordinary differential equation [9]. This makes tractable the study of the global asymptotic dynamics of the kinds of nonlinear integro-differential equations described above. This is the approach taken in this paper. In Sect. 2 the basic hierarchical model equations will be described and in Sect. 3 the intra-specific scramble and contest models are derived. The global asymptotic dynamics of the two types of models are described and compared, with regard to equilibrium levels and resilience, in Sect. 4 for both the case of a constant resource and a dynamically varying resource. Proofs of the results appear in the appendices.

\section{The model}

The continuous age structured model is formulated by means of the balance equation

$$
\lim _{h \rightarrow 0} \frac{\rho(t+h, a+h)-\rho(t, a)}{h}=-\delta \rho(t, a),
$$

where $\rho(t, a)$ is the per unit age density of organisms of age $a>0$ at time $t>0$ and $\delta \geqq 0$ is the per capita death rate $[26,31]$. Births are accounted for by the boundary condition

$$
\rho(t, 0)=\int_{0}^{\infty} \beta \rho(t, a) d a, \quad t>0
$$

where $\beta \geqq 0$ is the per capita birth rate. The model is completed by the prescription of an initial age distribution $\phi(a) \geqq 0$ and the requirement that

$$
\rho(0, a)=\phi(a), \quad a \geqq 0 .
$$


In general, the vital rates $\beta$ and $\delta$ are functions of time $t$ and age $a$. For density regulated populations they are also dependent on the distribution function $\rho$, usually by means of a linear functional of $\rho$. In the simplest density-dependent models $\beta$ and $\delta$ depend on total population size [16]

$$
P(t)=\int_{0}^{\infty} \rho(t, a) d a .
$$

Models involving more general weighted functions of $\rho$ were studied in [30]. A special kind of functional dependence of $\beta$ and $\delta$ on $\rho$ appropriate for age-specific hierarchies was introduced in [9], specifically

$$
\begin{gathered}
\beta=\beta(t, Y(t, a), O(t, a)) \\
\delta=\delta(t, Y(t, a), O(t, a)),
\end{gathered}
$$

where

$$
\begin{gathered}
Y(t, a)=\int_{0}^{a} \rho(t, \alpha) d \alpha \\
O(t, a)=\int_{a}^{\infty} \rho(t, \alpha) d \alpha .
\end{gathered}
$$

In these so-called "age hierarchical" models the vital rates $\beta$ and $\delta$ depend on time $t$, the number $Y(t, a)$ of individuals younger than age $a$, and the number $O(t, a)$ of individuals older than age $a$ (but do not otherwise depend explicitly on age $a$ ). This includes a possible dependence on total population size

$$
P(t)=Y(t, a)+O(t, a) .
$$

In [9] the existence and uniqueness of solutions of the nonlinear model equations (1)-(4) is rigorously addressed. In addition, it is shown in [9] that the dynamics of total population size $P(t)$ are governed by a scalar ordinary differential equation. This ordinary differential equation can be heuristically derived as follows. Under suitable conditions of smoothness, equation (1) can be written as

$$
\frac{\partial \rho}{\partial t}+\frac{\partial \rho}{\partial a}=-\delta \rho, \quad a>0, t>0 .
$$

Using the facts that $P=Y+O$ and

$$
\frac{\partial}{\partial a} Y(t, a)=\rho(t, a),
$$

one finds that

$$
\begin{aligned}
\rho(t, 0) & =\int_{0}^{\infty} \beta(t, Y(t, a), O(t, a)) \rho(t, a) d a \\
& =\int_{0}^{P} \beta(t, z, P-z) d z
\end{aligned}
$$


and

$$
\int_{0}^{\infty} \delta(t, Y(t, a), O(t, a)) \rho(t, a) d a=\int_{0}^{P} \delta(t, z, P-z) d z .
$$

These identities and an integration of the partial differential equation from $a=0$ to $a=\infty$ lead to the equation

$$
P^{\prime}(t)=B(t, P)-D(t, P)
$$

together with the initial condition

where

$$
P(0)=\int_{0}^{\infty} \phi(a) d a
$$

$$
\begin{aligned}
B(t, P) & =\int_{0}^{P} \beta(t, z, P-z) d z \\
D(t, P) & =\int_{0}^{P} \delta(t, z, P-z) d z .
\end{aligned}
$$

The study of the asymptotic dynamics of age hierarchical models is thus reduced to that of the scalar ordinary differential equation (5).

\section{Intra-specific competition}

In this section we will devise and study age hierarchical models in order to study two different modes of intra-specific competition for limiting resources, namely scramble and contest. The goal is to compare some of the dynamical consequences of these two modes of competition and to see in what sense one mode might be more advantageous to a population than the other.

Let $R$ denote the amount of a limiting resource available to the population. Let $c \in[0,1]$ denote the fraction of this amount that is available to an individual. In the presence of competition this fraction is dependent in some way on population density. We will consider model equations in which $c=c(Y, O)$ is a function of the functionals $Y$ and $O$. For such a case

$$
R c(Y(t, a), O(t, a))
$$

is the resource available to an individual of age $a$ at time $t$. The competition coefficient $c$ is assumed to satisfy the condition

$$
\int_{0}^{P} c(z, P-z) d z \leqq 1
$$

so that the total resource available to the whole population is less than $R$, i.e.

$$
\int_{0}^{\infty} R c(Y(t, a), O(t, a)) \rho(t, a) d a=\int_{0}^{P} R c(z, P-z) d z \leqq R .
$$


We assume the birth rate is proportional to a resource uptake rate

$$
u \in C^{2}\left(R^{+}, R^{+}\right), \quad u^{\prime}>0, u(0)=0
$$

so that

$$
\beta(Y, O)=\beta_{0} u(R c(Y, O)), \quad \beta_{0}>0 .
$$

The death rate is assumed to be constant:

$$
\delta=\delta_{0}>0 \text {. }
$$

We wish to compare the dynamics of scramble competition with those of contest intra-specific competition. To do this we construct models for each of two populations which are assumed to be identical in every way except in their mode of intra-specific competition, one of which is scramble and the other of which is contest. In so doing we utilize Lomnicki's definitions of scramble and contest competition [22]. According to this definition scramble competition occurs when every individual (potentially) affects the amount of resource available to any other individual in the population. On the other hand, contest competition occurs when no individual of age less than $a$ can affect the amount of resource available to an individual of age $a$. This leads us to assume in our model for scramble competition that the resource available to individuals is a function of the total population size $P$, whereas the resource available to individuals of age $a$ in our contest model is a function of the total number $O$ of individuals older than age $a$.

We consider competition coefficients $c=c(x)$ that satisfy the general conditions

$$
\begin{gathered}
c \in C^{1}\left(R^{+},[0,1]\right), \quad c(0)=1 \\
c^{\prime}<0, \quad \lim _{z \rightarrow \infty} c(z)=0,
\end{gathered}
$$

and denote the scramble competition coefficient by $c_{s}=c_{s}(x)$ and the contest competition coefficient by $c_{c}=c_{c}(x)$. The resource uptake rates for the scramble and contest populations are given by

$$
\begin{array}{ll}
\text { Scramble: } & u_{s}=u\left(R c_{s}(P)\right) \\
\text { Contest: } & u_{c}=u\left(R c_{c}(O)\right)
\end{array}
$$

where $u$ satisfies (6). We have assumed the functions $u, c_{s}$, and $c_{c}$ (and hence $u_{s}$ and $u_{c}$ ) do not explicitly depend on age $a$. Therefore, in the case of scramble competition all individuals in our model consume (per unit time) equal portions of resource, while in the case of contest competition all individuals in the same age class consume (per unit time) equal portions of resource.

In order to insure an appropriate comparison between the scramble and contest populations we impose the criterion that both modes of competition divide up the same amount of resource (for a given density distribution $\rho$ ). This requires a relationship between the competition coefficients $c_{s}$ and $c_{c}$ which is derivable from the equation

$$
\int_{0}^{\infty} R c_{s}(P(t)) \rho(t, a) d a=\int_{0}^{\infty} R c_{c}(O(t, a)) \rho(t, a) d a .
$$


If $R$ is independent of age $a$, it follows from this criterion that

$$
\int_{0}^{P} c_{s}(P) d z=\int_{0}^{P} c_{c}(z) d z
$$

or

$$
c_{s}(P) \doteq \frac{1}{P} \int_{0}^{P} c_{c}(z) d z .
$$

Thus, given a competition coefficient function

$$
c_{c}=c(O)
$$

for the contest population (where $c$ satisfies (7)), we consider, for comparison purposes, the competition coefficient function $c_{s}$ for the scramble population defined by (8).

For example, the pair

$$
c_{s}(P)=\frac{1}{1+P}, \quad c_{c}(O)=\frac{1}{(1+O)^{2}}
$$

satisfies the "comparison criterion" (8). In this example, every individual in the scramble population has available the same share $R /(1+P)$ of the resource and thus has an uptake rate of $u(R /(1+P))$ units of resource per unit time.

With these submodels for birth and death rates the models for both the scramble and contest populations are of the hierarchical form discussed in the previous section. The asymptotic dynamics of the models are therefore governed by scalar ordinary differential equations for total population size $P$. Specifically, the dynamics of the scramble and contest populations are determined by the equations

$$
\begin{array}{ll}
\text { Scramble: } & P^{\prime}=\beta_{0} u\left(R \frac{1}{P} \int_{0}^{P} c(z) d z\right) P-\delta_{0} P \\
\text { Contest: } & P^{\prime}=\beta_{0} \int_{0}^{P} u(R c(z)) d z-\delta_{0} P .
\end{array}
$$

Defining the number

$$
n=\frac{\beta_{0} u(R)}{\delta_{0}},
$$

we normalize the model equations as

$$
\begin{array}{ll}
\text { Scramble: } & P^{\prime}=\delta_{0}\left[\frac{n}{u(R)} u\left(\frac{1}{P} \int_{0}^{P} R c(z) d z\right)-1\right] P \\
\text { Contest: } & P^{\prime}=\delta_{0}\left[\frac{n}{u(R)} \frac{1}{P} \int_{0}^{P} u(R c(z)) d z-1\right] P .
\end{array}
$$

The number $n$ is the "inherent net reproductive number". It is the expected number of offspring per individual per life time at low population densities. 


\section{Results}

In this section we will compare some general conclusions about the dynamics implied by the scramble and contest models (9) and (10). We do this first for the case when the resource level $R$ is constant in time.

\subsection{Constant $R$}

When $R$ is constant the following theorem is proved in the Appendix for both equations (9) and (10):

Theorem 1. If $n<1$, then $\lim _{t \rightarrow \infty} P(t)=0$. If $n>1$, then the trivial equilibrium $P=0$ is unstable and there exists a unique positive equilibrium $P_{\infty}$ which is globally asymptotically stable.

For $n>1$ denote the equilibria of equations (9) and (10) by $P_{s}$ and $P_{c}$ respectively.

4.1.1 Comparison of the equilibria $\mathrm{P}_{s}$ and $\mathrm{P}_{c}$

Suppose $u^{\prime \prime}<0$ on $(0, R)$. By Jensen's Inequality, a form of which is proved in the Appendix, it follows that

$$
\frac{1}{P} \int_{0}^{P} u(R c(z)) d z<u\left(\frac{1}{P} \int_{0}^{P} R c(z) d z\right)
$$

for all $P>0$. At the scramble and contest equilibria $P_{s}$ and $P_{c}$ we have

Since both

$$
\frac{1}{P_{c}} \int_{0}^{P_{c}} u(R c(z)) d z=\frac{u(R)}{n}=u\left(\frac{1}{P_{s}} \int_{0}^{P_{s}} R c(z) d z\right) .
$$

$$
\frac{1}{P} \int_{0}^{P} u(R c(z)) d z \text { and } u\left(\frac{1}{P} \int_{0}^{P} R c(z) d z\right)
$$

are decreasing functions of $P$, it follows that $P_{c}<P_{s}$.

A similar argument shows that if $u^{\prime \prime}>0$ on $(0, R)$, then $P_{s}<P_{c}$.

Theorem 2. Let $n>1$. If $u^{\prime \prime}<0$ on $(0, R)$, then $P_{c}<P_{s}$. If $u^{\prime \prime}>0$ on $(0, R)$, then $P_{s}<P_{c}$.

This theorem asserts that a concave down $u$ implies the scramble population has a larger equilibrium, while a concave up $u$ implies the contest population has a larger equilibrium.

For example, if $u$ is a Holling II hyperbolic functional response (and hence always concave down), the scramble population will have the larger equilibrium.

An S-shaped Holling III type functional response $u$ has a change of concavity. In this case $u$ will be concave up on $(0, R)$ for sufficiently low values of resource $R$ and so the contest population will have the larger equilibrium. 
On the other hand, for large values of $R$ the response functional $u$ changes concavity from up to down on the interval $(0, R)$. In this case, the following example illustrates that it is possible for the scramble population to have the larger equilibrium for sufficiently large $R$. If we take

$$
u(R)=\frac{R^{2}}{k^{2}+R^{2}} \quad \text { and } \quad c(z)=\frac{1}{(1+z)^{2}}
$$

then the scramble equilibrium is given by

$$
P_{s}=\frac{R}{k} \sqrt{\frac{\beta}{\delta}-1}-1 .
$$

The contest equilibrium is a root of the equation

$$
P=\frac{\beta}{\delta} \sqrt{\frac{R}{k}} \int_{\sqrt{k / R}}^{(1+P) \sqrt{k / R}} \frac{1}{1+w^{4}} d w
$$

Therefore, $0<P_{c} \leqq m \sqrt{R}$ for some constant $m>0$. It follows that $0<P_{c}<P_{s}$ for sufficiently large $R$.

Thus, with respect to equilibria levels, an S-shaped uptake rate implies contest competition is more advantageous for low resource levels, while scramble competition can be more advantageous for high resource levels.

\subsubsection{Comparison of equilibrium resilience}

We next compare the resilience of the scramble and contest equilibria. If a population is perturbed away from equilibrium by $\Delta P$, then the resilience is the magnitude of the linearized eigenvalue

$$
\frac{1}{\Delta P} \frac{d(\Delta P)}{d t} \approx \frac{d P^{\prime}}{d P}
$$

evaluated at the equilibrium. If $\lambda_{s}$ and $\lambda_{c}$ denote the resilience $d P^{\prime} / d P$ of the scramble and contest populations, respectively, at their equilibria $P_{s}$ and $P_{c}$, then a straightforward calculation shows that

$$
\begin{aligned}
\lambda_{s}-\lambda_{c}= & \frac{\delta_{0} n}{u(R)}\left[u^{\prime}\left(\frac{1}{P_{s}} \int_{0}^{P_{s}} R c(z) d z\right)\left(R c\left(P_{s}\right)-\frac{1}{P_{s}} \int_{0}^{P_{s}} R c(z) d z\right)\right. \\
& \left.+u\left(\frac{1}{P_{s}} \int_{0}^{P_{s}} R c(z) d z\right)-u\left(R c\left(P_{c}\right)\right)\right] .
\end{aligned}
$$

We must therefore compare linearization of $u$ at $P_{s}^{-1} \int_{0}^{P_{s}} R c(z) d z$ evaluated at $R c\left(P_{s}\right)$ with the value of $u$ at $R c\left(P_{c}\right)$.

The proof of the following theorem is indicated in the Appendix.

Theorem 3. Let $n>1$. If $u^{\prime \prime}(R)<0$, then $\lambda_{c}<\lambda_{s}$ whenever $n$ is sufficiently close to 1 . If $u^{\prime \prime}(R)>0$, then $\lambda_{s}<\lambda_{c}$ whenever $n$ is sufficiently close to 1 . 
Since the positive equilibria $P_{s}$ and $P_{c}$ are asymptotically stable, we know that $\lambda_{s}<0$ and $\lambda_{c}<0$. Therefore, this theorem asserts that, when $n$ is close to 1 , a concave down $u$ implies the contest population is more resilient, while a concave up $u$ implies the scramble population is more resilient.

If greater resilience and larger equilibrium levels are considered advantageous, then Theorems 2 and 3 imply a trade off with regard to which competitive mode, scramble or contest, is more advantageous. The resilience criterion gives roughly opposite conclusions from the equilibria level criterion, at least for $n$ close to 1 .

\subsubsection{Comparison of asymptotic age distributions}

So far we have compared scramble and contest competition by using total numbers of individuals in a population. It is also reasonable to ask how the mode of competition affects the stationary age distributions at the positive equilibria $P=P_{s}$ and $P=P_{c}$. In general,

$$
\begin{aligned}
\rho(t, a)= & B(t-a, P(t-a)) \\
& \times \exp \left(-\int_{0}^{a} \delta(t-a+\alpha, Y(t-a+\alpha, \alpha), O(t-a+\alpha, \alpha)) d \alpha\right)
\end{aligned}
$$

for $t>a[20]$. At equilibrium we have

and therefore

$$
B(P)=D(P)=\delta_{0} P
$$

$$
\lim _{t \rightarrow \infty} \rho(t, a)=\delta_{0} P \mathrm{e}^{-\delta_{0} a} .
$$

The scramble and contest stationary age distributions are therefore

$$
\delta_{0} P_{s} \mathrm{e}^{-\delta_{0} a} \text { and } \delta_{0} P_{c} \mathrm{e}^{-\delta_{0} a}
$$

respectively. This shows that for the models considered here whatever relationship is borne between the scramble $P_{s}$ and contest $P_{c}$ total population levels also holds for every age class.

\subsection{Dynamic $R$}

We now consider a model in which $R$ is not constant in time as was the case in the previous models. Specifically, in the absence of consumption by individuals of the biological population, it is assumed the dynamics of $R$ are governed by an equation $R^{\prime}=I(R)$ where $I(R)$ models an influx of $R$ into the environment. In the presence of the population, the dynamics of $R$ are governed by an equation of the form

$$
R^{\prime}(t)=I(R)-F(R, P),
$$

where $F(R, P)$ describes the resource consumption rate. We will examine the prototypical example $I(R)=r\left(R_{0}-R\right)$ for which, in the absence of 
consumption by the population, $R$ will equilibrate to a level $R_{0}>0$. The coefficient $r$ is a measure of the rate at which this equilibration takes place.

Rescaling resource units and time we can, without loss in generality, assume that $I(R)=1-R$. We then arrive the following two models for scramble and contest competition respectively:

$$
\begin{aligned}
& \left\{\begin{array}{l}
R^{\prime}=1-R-P u\left(R \frac{1}{P} \int_{0}^{P} c(z) d z\right) \\
P^{\prime}=\beta_{0} P u\left(R \frac{1}{P} \int_{0}^{P} c(z) d z\right)-\delta_{0} P
\end{array}\right. \\
& \left\{\begin{array}{l}
R^{\prime}=1-R-P \frac{1}{P} \int_{0}^{P} u(R c(z)) d z \\
P^{\prime}=\beta_{0} P \frac{1}{P} \int_{0}^{P} u(R c(z)) d z-\delta_{0} P .
\end{array}\right.
\end{aligned}
$$

The proof of the following theorem is outlined in the Appendix for both models, where the net reproduction number is defined to be $n=\beta_{0} u(1) / \delta_{0}$ :

Theorem 4. If $n<1$, the axis equilibrium $(R, P)=(1,0)$ is globally asymptotically stable. If $n>1,(R, P)=(1,0)$ is unstable and there exists a unique positive equilibrium $\left(R_{\infty}, P_{\infty}\right) \in(0,1) \times(0, n / u(1))$ which is globally asymptotically stable.

In order to compare the positive equilibrium levels $P_{s}$ and $P_{c}$ for the scramble and contest populations, we note that both

and

$$
u\left(\left(1-P \frac{u(1)}{n}\right) \frac{1}{P} \int_{0}^{P} c(z) d z\right)
$$

$$
\frac{1}{P} \int_{0}^{P} u\left(\left(1-P \frac{u(1)}{n}\right) c(z)\right) d z
$$

are decreasing functions of $P$ on the interval $(0, n / u(1))$ (see the Appendix). Moreover, by Jensen's Inequality (as proved in the Appendix), the first expression is the larger for all $P \in(0, n / u(1))$ when $u(\cdot)$ is concave down on $(0,1)$ and the second is the larger when $u(\cdot)$ is concave up on $(0,1)$. Since at equilibrium

$$
u\left(\left(1-P_{s} \frac{u(1)}{n}\right) \frac{1}{P_{s}} \int_{0}^{P_{s}} c(z) d z\right)=\frac{u(1)}{n}=\frac{1}{P_{c}} \int_{0}^{P_{c}} u\left(\left(1-P_{c} \frac{u(1)}{n}\right) c(z)\right) d z
$$

we have the same result obained for the case of constant $R$ :

Theorem 5. Let $n>1$. If $u^{\prime \prime}<0$ on $(0,1)$, then $P_{c}<P_{s}$. If $u^{\prime \prime}>0$ on $(0,1)$, then $P_{s}<P_{c}$. In any case, $\left(P_{s}-P_{c}\right)\left(R_{c}-R_{s}\right)>0$. 
A corollary of this result is that the mode of intra-specific competition which results in the larger population equilibrium also exploits the resource to the lower equilibrium level.

\section{Generalizations and further modifications}

In general, one might ask if the original partial differential equation model can be reduced to an ordinary differential equation when the hierarchy is based on some variable other than age. In the following, we indicate the procedure for a hierarchy based on size $m$ of the individual. For related details see $[9,8$, $10,34]$.

In this case, the structured partial differential equation model is

$$
\begin{gathered}
\frac{\partial \rho}{\partial t}+\frac{\partial}{\partial m}(\gamma \rho)=-\delta \rho \\
\left.\gamma \rho\right|_{m=m_{b}}=\int_{m_{b}}^{\infty} \beta \rho(t, m) d m \\
\rho(0, m)=\phi(m),
\end{gathered}
$$

where

$$
\frac{d m}{d t}=\gamma(t, m, \rho)
$$

is the growth rate along characteristics of the mass variable $m$.

We assume the competition coefficient $c=c(t, S, L)$ for an individual of mass $m$ depends on $S$, the total biomass of individuals of smaller size, and $L$, the total biomass of indivduals of larger size, where

$$
\begin{aligned}
S(t, m) & =\int_{m_{b}}^{m} s \rho(t, s) d s \\
L(t, m) & =\int_{m}^{\infty} s \rho(t, s) d s .
\end{aligned}
$$

If we assume that the birth and growth rates are proportional to body size and are functions of $S$ and $L$

$$
\begin{gathered}
\beta(t, m, \rho)=m b(t, S, L) \\
\gamma(t, m, \rho)=m g(t, S, L),
\end{gathered}
$$

then an ordinary differential equation for total population biomass

$$
P=S+L=\int_{m_{b}}^{\infty} s \rho(t, s) d s
$$

can be obtained by multiplying the partial differential equation (11) by $m$ and integrating from $m=m_{b}$ to $m=\infty$. Under the additional assumption that the 
per unit death rate $\delta$ is a function of $S$ and $L$

$$
\delta=\delta(t, S, L)
$$

$\left(\right.$ and $\left.\lim _{m \rightarrow \infty}(m \rho g)=0\right)$ this integration results in the scalar ordinary differential equation

$$
P^{\prime}(t)=B(t, P)+G(t, P)-D(t, P)
$$

for the total biomass $P$ with the initial condition

where

$$
P(0)=\int_{m_{b}}^{\infty} m \phi(m) d m,
$$

$$
\begin{aligned}
& B(t, P)=m_{b} \int_{0}^{P} b(t, z, P-z) d z \\
& G(t, P)=\int_{0}^{P} g(t, z, P-z) d z \\
& D(t, P)=\int_{0}^{P} \delta(t, z, P-z) d z .
\end{aligned}
$$

The scalar equation (12), which contains an added term $G(t, P)$ that accounts for growth, can be compared to the corresponding equation (5) for age-structured populations (in which only birth and death rates appear). If, following the modeling methodology above for the age-structured case, we assume that both (per unit size) birth and growth rates are proportional to resource uptake

$$
\begin{aligned}
b(S, L)=\beta_{0} u(R c(S, L)), & \beta_{0}>0 \\
g(S, L)=\gamma_{0} u(R c(S, L)), & \gamma_{0}>0
\end{aligned}
$$

and that the death rate is a constant $\delta_{0}>0$, then the equation (12) for total population biomass $P$ takes exactly the same form as those studied above for the age-structured case (with $\beta_{0}$ replaced now by $m_{b} \beta_{0}+\gamma_{0}$ ). We conclude that all of our results concerning the comparison between scramble and contest competitive modes remain valid for this size-structured model.

\section{Concluding remarks}

Utilizing the definitions of scramble and contest competition in [22] we derived models for the dynamics of populations practicing these types of intra-specific competition from the hierarchical models of the form (1)-(4). From the results of [9] and the comparison criterion (8), the scalar ordinary differential equations (10) and (9) for the dynamics of total population sizes of the scramble and contest populations were obtained. Conclusions concerning the comparative advantages and disadvantages of these two modes of competition can therefore be drawn from a study of the asymptotic dynamics of these two equations. Both models predict population extinction if the inherent 
net reproductive number $n$ is less than 1 and population survival (in the form of a globally asymptotically stable positive equilibrium) if $n$ is greater than 1 .

With regard to the relative sizes of positive equilibria (when $n>1$ ) the concavity of the resource uptake rate $u$, as a function of the available resource, is a crucial factor. If $u$ is concave down then the scramble population will have the larger equilibrium. This occurs, for example, with Holling type II (or Michaelis-Menton) uptake rates. This case is in disagreement with Lomincki's tenet that contest competition is more advantageous to the population. If, however, $u$ is concave up then the reverse is true, namely the contest population will have the larger equilibrium, in agreement with Lominicki's tenet. This case occurs, for example, with a Holling type III resource uptake rate at "low" resource availability rates. These conclusions were shown to remain valid for a dynamically varying resource $R$.

A comparison between scramble and contest competition was made with respect to the resilience of the equilibrium, at least for $n>1$ values not too large. Here it is again found that the concavity of the uptake rate $u$ is important. However, the opposite conclusions are reached from those reached when the equilibrium level is used to make the comparison. Namely, if $u$ is concave down then the contest population is more resilient (although it has the smaller equilibrium size), in agreement with Lominicki's tenet, while the reverse is true if $u$ is concave up and resource levels are low.

The conclusions above were shown to remain valid for a general class of size-structured, continuous population models (as opposed to age-structured). Discrete hierarchical models are studied in [35]. In this reference it is shown that the dynamics of total population size, in analogy to (5), are governed by a one dimensional map. This methodology is used to study several applications, including the kinds of competition interactions studied in this paper.

We conclude that the general tenet that contest competition is more advantageous than scramble competition needs to be carefully qualified. First of all there is the rather obvious warning that the meaning of "advantageous" must be made clear and how the comparison is to be made must be specified. Furthermore, as we have seen, certain nonlinear details can be a determining factor in the comparison. In our case, this was the resource uptake rate. Other factors might prove to be important in other models. For example, we ignored in this paper the effects of the resource competition on individual survival rates. What role such nonlinearities might play in comparing the two modes of competition remains to be studied.

\section{A Appendix}

\section{A.1 Proof of Therem 1}

Since $u(0)=0, u^{\prime}>0, c(0)=1, c^{\prime}<0$, and $\lim _{z \rightarrow \infty} c(z)=0$, the functions

$$
u\left(R \frac{1}{P} \int_{0}^{P} c(z) d z\right)
$$


and

$$
\frac{1}{P} \int_{0}^{P} u(R c(z)) d z
$$

both approach $u(R)$ as $P \rightarrow 0$, approach 0 as $P \rightarrow \infty$, and decrease monotonically in $P$. Therefore, for each model, if $n<1, P^{\prime}=0$ if and only if $P=0$, and $P^{\prime}<0$ for all $P>0$. Furthermore, if $n>1$, then for each model there is a unique positive equilibrium $P_{\infty}$, and $P^{\prime}<0$ whenever $P>P_{\infty}$ and $P^{\prime}>0$ whenever $0<P<P_{\infty}$.

\section{A.2 Proof of a Jensen-type inequlity}

The following theorem is a form Jensen's Inequality:

Theorem 6. Suppose $u \in C^{2}[0, \infty)$ and $f:(0, \infty) \times[0, \infty) \rightarrow R$ is continuous. If there exists a $c>0$ such that $u^{\prime \prime}(f(x, y))<0$ on $(0, c) \times(0, x)$ and $f=f(x, y)$ is monotonic in $y$ on $(0, x)$ for each $x \in(0, c)$, then

for all $x \in(0, c)$.

$$
\frac{1}{x} \int_{0}^{x} u(f(x, z)) d z<u\left(\frac{1}{x} \int_{0}^{x} f(x, z) d z\right)
$$

Proof. If $u^{\prime \prime}(x)<0$ on $(a, b)$, then for all $x \in[a, b]$ and $y \in(a, b)$,

$$
u(x) \leqq u^{\prime}(y)(x-y)+u(y)
$$

with the strict inequality holding everywhere except at $x=y$.

Let $x \in(0, c)$. For all $y \in[0, x]$

$$
u(f(x, y)) \leqq u^{\prime}\left(\frac{1}{x} \int_{0}^{x} f(x, z) d z\right)\left\{f(x, y)-\frac{1}{x} \int_{0}^{x} f(x, z) d z\right\}+u\left(\frac{1}{x} \int_{0}^{x} f(x, z) d z\right)
$$

with the strict inequality holding everywhere except at the unique $y \in(0, x)$ where

$$
f(x, y)=\frac{1}{x} \int_{0}^{x} f(x, z) d z .
$$

Integration with respect to $y$ of both sides of the inequality yields the conclusion.

The analogous theorem with the inequalities reversed can be similarly proved.

\section{A.3 Proof of Theorem 3}

It is sufficient to compare

$$
\tilde{\lambda}_{s}=u^{\prime}\left(\frac{1}{P_{s}} \int_{0}^{P_{s}} R c(z) d z\right)\left\{R c\left(P_{s}\right)-\frac{1}{P_{s}} \int_{0}^{P_{s}} R c(z) d z\right\}+u\left(\frac{1}{P_{s}} \int_{0}^{P_{s}} R c(z) d z\right)
$$


and

$$
\tilde{\lambda}_{c}=u\left(R c\left(P_{c}\right)\right) .
$$

When $n=1, P_{s}=P_{c}=0$ and $\tilde{\lambda}_{s}=u(R)$, so for $n$ sufficiently close to 1 , $\tilde{\lambda}_{s}>0$. Thus,

since

$$
u(0)=0<\tilde{\lambda}_{s}<u\left(\frac{1}{P_{s}} \int_{0}^{P_{s}} R c(z) d z\right)
$$

Also $u^{\prime}>0$, so

$$
R c\left(P_{s}\right)<\frac{1}{P_{s}} \int_{0}^{P_{s}} R c(z) d z
$$

$$
Q(n) \doteq u^{-1}\left(\tilde{\lambda}_{s}(n)\right)
$$

is well-defined and is a continuous function of $n$.

Expanding $P_{s}(n), P_{c}(n), c\left(P_{c}(n)\right)$, and $Q(n)$ in a Taylor series about $n=1$, we find

to second order.

$$
Q(n)-R c\left(P_{c}(n)\right) \approx \frac{1}{2}\left[\frac{-u^{\prime \prime}(R)(u(R))^{2}}{3\left(u^{\prime}(R)\right)^{3}}\right](n-1)^{2}
$$

If $u^{\prime \prime}(R)<0$, then $R c\left(P_{c}\right)<Q$ when $n$ is sufficiently close to 1 and so

$$
\tilde{\lambda}_{c}=u\left(R c\left(P_{c}\right)\right)<u(Q)=\tilde{\lambda}_{s} .
$$

If $u^{\prime \prime}(R)>0$, then $Q<R c\left(P_{c}\right)$ when $n$ is sufficiently close to 1 and so

$$
\tilde{\lambda}_{c}=u(Q)<u\left(R c\left(P_{c}\right)\right)=\tilde{\lambda}_{s} .
$$

\section{A.4 Proof of Theorem 4}

As in [9] one can prove that solutions to both models are bounded by showing each triangle $0 \leqq R \leqq k, 0 \leqq P \leqq \beta_{0}(k-R)$ is positively invariant for $k>\max \left\{1,1 / \delta_{0}\right\}$.

Each model has an axis equilibrium $(1,0)$. Any interior equilibria must satisfy the equations

$$
f_{s}(P) \doteq u\left(\left(1-P \frac{u(1)}{n}\right) \frac{1}{P} \int_{0}^{P} c(z) d z\right)=\frac{u(1)}{n}
$$

for the scramble model and

$$
f_{c}(P) \doteq \frac{1}{P} \int_{0}^{P} u\left(\left(1-P \frac{u(1)}{n}\right) c(z)\right) d z=\frac{u(1)}{n}
$$

for the contest model.

Clearly

$$
f_{s}\left(\frac{n}{u(1)}\right)=f_{c}\left(\frac{n}{u(1)}\right)=0 .
$$


Also, $f_{s}(P)$ approaches $u(1)$ as $P \rightarrow 0$ and is monotonically decreasing in $P$ on $(0, n / u(1))$.

From

$$
\begin{aligned}
& \frac{d}{d P} \int_{0}^{P} u\left(\left(1-P \frac{u(1)}{n}\right) c(z)\right) d z \\
& \quad=-\frac{u(1)}{n} \int_{0}^{P} u^{\prime}\left(\left(1-P \frac{u(1)}{n}\right) c(z)\right) c(z) d z+u\left(\left(1-P \frac{u(1)}{n}\right) c(P)\right)
\end{aligned}
$$

we can show, using l'Hopital's rule, that $f_{c}(P)$ approaches $u(1)$ as $P \rightarrow 0$. From

$$
\begin{aligned}
\frac{d f_{c}}{d P}= & -\frac{u(1)}{n P} \int_{0}^{P} u^{\prime}\left(\left(1-P \frac{u(1)}{n}\right) c(z)\right) c(z) d z \\
& +\frac{1}{P}\left[u\left(\left(1-P \frac{u(1)}{n}\right) c(P)\right)-\frac{1}{P} \int_{0}^{P} u\left(\left(1-P \frac{u(1)}{n}\right) c(z)\right) d z\right]
\end{aligned}
$$

it follows that $f_{c}$ is monotonically decreasing in $P$ on the interval $(0, n / u(1))$.

Thus, each model has an interior equilibrium if and only if $n>1$, and this equilibrium is unique.

A straightforward linearlized stability analysis for each model shows the axis equilibrium $(R, P)=(1,0)$ is stable when $n<1$ and is a saddle when $n>1$, with its stable manifold on the $R$ axis. In the latter case, the HartmanGrobman Theorem implies $(1,0)$ cannot be contained in the $\omega$-limit set of a positive orbit. An application of the Dulac-Bendixson Theorem (with factor $1 / P$ ) to each model shows there are no closed orbits. By Poincare-Bendixson theory the $\omega$-limit set of any positive orbit must in fact be the interior equilibrium.

\section{References}

1. Botsford, L. W., The effects of increased individual growth rates on depressed population size, Am. Nat. 117: 38-63 (1981)

2. Busenberg, S. and M. Iannelli, Separable models in age-dependent population dynamics, J. Math. Biol. 22: 145-173 (1985)

3. Costantino, R. F., and R. A. Desharnais, Population Dynamics and the Tribolium Model: Genetics and Demography, Monographs on Theoretical and Applied Genetics 13, Springer, Berlin, 1991

4. Cushing, J. M. and Jia Li, On Ebenman's model for the dynamics of a population with competing juveniles and adults, Bull. Math. Biol. 51: 687-713 (1989)

5. Cushing, J. M. and Jia Li, Juvenile versus adult competition, J. Math. Biol. 29: 457-473 (1991)

6. Cushing, J. M. and Jia Li, Intra-specific competition and density dependent juvenile growth, Bull. Math. Biol. 54(4): 503-519 (1992)

7. Cushing, J. M., A simple model of cannibalism, Math. Biosci. 107: 47-71 (1991)

8. Cushing, J. M., A size-structured model for cannibalism, Theor. Popul Biol. 42(3), 347-361 (1992) 
9. Cushing, J. M., The dynamics of hierarchical age-structured populations, J. Math. Biol. 32: 705-729 (1994)

10. Cushing, J. M., Competition in hierarchically size-structured populations, to appear, Proceedings International Conference on Differential Equations \& Applications to Biology and Industry, World Scientific Publ., Co., Singapore, 1995

11. Diekmann, O., R. M. Nisbet, W. S. C. Gurney and F. van den Bosch, Simple mathematical models for cannibalism: a critique and a new approach, Math. Biosci. 78: $21-46$ (1986)

12. Ebenman, B., Niche differences between age classes and intra-specific competition in age-structured populations, J. Thor. Biol. 124: 25-33 (1987)

13. Ebenman, B., Competition between age classes and population dynamics, J. Theor. Biol. 131: 389-400 (1988)

14. Ebenman, B., Dynamics of age- and size-structured populations: intra-specific competition, Size-structured Populations (Ebenman \& Persson eds.), Springer-Verlag, Berlin, 127-139, 1988

15. Ebenman, Bo and Lennart Persson, Size-structured Populations: Ecology and Evolution, Springer, Berlin, 1988

16. Gurtin, M. and R. C. MacCamy, Nonlinear age dependent population dynamics, Arch. Rat. Mech. Anal. 54: 281-300 (1974)

17. Gurtin, M. and R. C. MacCamy, Some simple models for nonlinear age-dependent population dynamics, Math. Biosci. 43: 199-211 (1979)

18. Hastings, A., Cycles in cannibalistic egg-larval interactions, J. Math. Biol. 24: 651-666 (1987)

19. Hastings, A. and R. F. Costantino, Cannibalistic egg-larva interactions in Tribolium: An explanation for the oscillations in population numbers, Am. Nat. 130: 36-52 (1987)

20. Hoppenstaedt, Frank, Mathematical Theories of Populations: Deomographics, Genetics and Epidemics, Reg. Conf. Series in Appl. Math., SIAM, Philadelphia, 1975

21. Landahl, H. D. and B. D. Hansen, A three stage population model with cannibalism, Bull. Math. Biol. 37: 11-17 (1975)

22. Lomnicki, A., Population Ecology of Individuals, Monographs in Population Biology 25, Princeton University Press, Princeton, New Jersey, 1988

23. Lomnicki, A. and J. Ombach: Resource partitioning within a single species population and population stability: a theoretical model, Theor. Pop. Biol. 25, 21-28 (1984)

24. Lomnicki, Adam and Stanislaw Sedziwy, Resource partitioning and population stability under exploitation competition, J. Theor. Biol. 132,: 119-120 (1988)

25. Loreau, M., Competition between age classes, and the stability of stage-structured populations: a re-examination of Ebenman's model, J. Theor. Biol. 144: 567-571 (1990)

26. Metz, J. A. J. and O. Diekmann, The Dynamics of Physiologically Structured Populations, Lecture Notes in Biomathematics 68, Springer, Berlin, Heidelberg, New York, 1986

27. Rogers, Alan R., Population dynamics under exploitation competition, J. Theor. Biol. 119: 363-368 (1986)

28. Stephen D. Simmes, Age dependent population dynamics with nonlinear interactions in the death rate, Ph.D. dissertation, Carnegie-Mellon University, 1978

29. Tschumy, W. O.: Competition between juveniles and adults in age-structured populations, Theor. Pop. Biol. 21: 255-268 (1982)

30. Tucker, S. L. and S. O. Zimmerman, A nonlinear model of population dynamics containing an arbitrary number of continuous structure variables, SIAM J. Appl. Math. 48(3), 549-591 (1988)

31. Webb, G. F. Theory of Nonlinear Age-Dependent Population Dynamics, Marcel Dekker, Inc., New York 1985

32. Werner, E. E. and J. F. Gilliam, The onogenetic niche and species interactions in size-structured populations, Ann. Rev. Ecol. Syst. 15, 393-425 (1984)

33. Van den Bosch, F., A. M. de Roos and W. Gabriel, Cannibalism as a life boat mechanism, J. Math. Biol. 26: 619-693 (1988) 